\title{
AMNESIE ET AUTRES EFFETS DU DIAZEPAM DANS LA PREMEDICATION ET L'INDUCTION DE L'ANESTHESIE
}

\author{
L. Authier, ${ }^{*} \ddagger$ R. Chapados, ${ }^{*}$ A. Sindon* et M.-A. Gagnon†
}

EN ADMINISTRATION intraveineuse, le diazépam est un inducteur anesthésique qui modifie très peu les grandes fonctions physiologiques ${ }^{1-s}$ et qui, contrairement au thiopental, n'entraîne pas d'excitation du système nerveux central. ${ }^{6} \mathrm{Par}$ ailleurs, la perte du réflexe ciliaire est moins rapide et malgré cette perte, certains sujets conservent même la capacité de répondre à un ordre verbal simple.

Ce décalage tempore] marqué entre la dépression de l'activité réflexe et celle de l'activité mentale enlève au critère habituel de l'induction anesthésique la sécurité qu'on lui accorde. En effet, après l'abolition du réflexe ciliaire, le patient est peut-être encore conscient. Le cas échéant, il pourrait y avoir mémorisation de la curarisation, de la laryngoscopie et de l'intubation. C'est pourquoi il est souhaitable qu'un agent inducteur tel que le diazépam produise une amnésie antérograde marquée. Il paraît cependant préférable que l'amnésie s'étende aussi à la phase qui précède immédiatement l'induction, puisqu'elle n'est pas dépourvue d'événements anxiogènes. Cette phase pourrait être touchée de façon antérograde par la prémédication, et aussi, de façon rétrograde, par l'agent d'induction luimềme.

Dans cette étude, nous avons voulu estimer les effets amnésiques du diazépam utilisé tant en prémédication qu'à l'induction. Le diazépam est comparé à la mépéridine en prémédication et au thiopental à l'induction. L'amnésie, les pertubations accompagnant l'induction, le temps d'éveil et les effets subjectifs post-anesthésiques sont mesurés avec les quatre combinaisons possibles des agents d'induction et de prémédication. De plus, l'analyse des effets sur ces différentes variables tient compte de la présence ou de l'absence de l'intubation.

METHodes
Sujets
Quarante volontaires, dont 32 de sexe fêminin
*Hôpital Maisonneuve-Rosemont.
†Hôpital Louis-H. Lafontaine et INRS-Santé.
†Demandes de tirés à part: Dr. L. Authier, Départe-
ment d'anesthésie, Hôpital Notre-Dame, 1560 rue
Sherbrooke Est, Montréal, Québec, Canada, H2L 4MI.

Canad. Anaesth. Soc. J., vol. 25, no. 4, July 1978 et 8 de sexe masculin, ont participé à l'expérience. Leur âge se situait entre 20 et 46 ans (moyenne: 32.6 ) et leur poids entre 48 et $96 \mathrm{~kg}$ (moyenne: 61.8). Le risque anesthésique des patients n'a pas dépassé le grade I. Ils étaient soumis à une intervention chirurgicale élective mineure: presque tous des cas de gynécologie (ligature de trompes) ou d'orthopédie (cure d'hallus valgus, par exemple). Une mauvaise physiologie de l'ceil ou de l'oreille, de même qu'une intervention sur ces organes, constituajent des critères d'exclusion.

\section{Médicaments}

En prémédication, tous les sujets ont reçu 30 mg de flurazépam per os au coucher la veille de l'intervention. A l'appel, ils recevaient tous, par la voie intramusculaire, $0.4 \mathrm{mg}$ d'atropine avec soit $1 \mathrm{mg} / \mathrm{kg}$ de mépéridine, soit $0.15 \mathrm{mg} / \mathrm{kg}$ de diazépam, selon le schéma expérimental.

Avant l'induction anesthésique, une canule no 18 munie d'un robinet à trois entrées était mise en place dans une veine de l'avant-bras; une des entrées a servi à l'infusion de soluté dextrosé (5 pour cent) - $\frac{1}{d}$ salin, à raison de $125 \mathrm{ml} / \mathrm{m}^{2}$ / heure, additionné durant la première heure d'anesthésie d'une quantité de $75 \mathrm{ml}$ pour chaque heure écoulée depuis 8:00h.

Par une autre entrée de la canule, on infusait jusqu'à perte du réflexe ciliaire l'agent d'induction; soit le diazépam, à raison de $3 \mathrm{mg} / 10 \mathrm{sec}$, soit le thiopental, à raison de $25 \mathrm{mg} / 10 \mathrm{sec}$, selon le plan expérimental.

La moitié des sujets furent intubés et ont reçu 1 $\mathrm{mg} / \mathrm{kg}$ de succinylcholine par la même canule. L'anesthésie de tous les sujets fut maintenue par l'halothane (entre 0.5 et 1.5 pour cent) dans un mélange $4 / 2$ de $\mathrm{N}_{2} \mathrm{O} / \mathrm{O}_{2}$. Aucun autre médicament ne fut utilisé jusqu'à l'éveil des patients.

Mesures
L'anmésie est évaluée selon une technique
déjà décrite ${ }^{7}$ qui comporte un questionnaire
mesurant la rétention mnémonique d'un stimulus
auditif présenté au sujet immédiatement avant
l'induction anesthésique. A l'aide d'écouteurs, le
sujet entend une mélodie facilement identifiable,
une sonnerie, suivie d'un texte de réclame pub297 
TABLEAU I

\begin{tabular}{|c|c|c|}
\hline \multicolumn{2}{|c|}{$\begin{array}{l}\text { Questionnaire } \\
\text { Date du questionnaire: } \\
\text { Heure du questionnaire: }\end{array}$} & Points \\
\hline \multicolumn{2}{|r|}{$\begin{array}{l}\text { 1) Dans les quelques minutes qui ont précédé l'anesthésie, vous } \\
\text { souvenez-vous d'avoir entendu une partie d'une émission de radio? } \\
\text { oui }\end{array}$} & 1 \\
\hline \multirow{2}{*}{\multicolumn{2}{|c|}{ 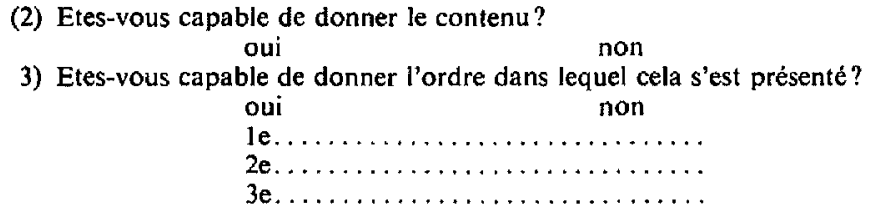 }} & 3 \\
\hline & & 2 \\
\hline \multicolumn{2}{|r|}{$\begin{array}{l}\text { (4) Etes-vous capable d'identifier la musique que vous avez entendue? } \\
\text { oui non }\end{array}$} & 1 \\
\hline \multirow{3}{*}{\multicolumn{2}{|c|}{$\begin{array}{l}\text { (5) Etes-vous capable d'identifier le bruit? } \\
\text { oui } \\
\text { (6) Etes-vous capable de raconter la discussion? } \\
\text { oui }\end{array}$}} & 1 \\
\hline & & 1 \\
\hline & & 9 \\
\hline
\end{tabular}

licitaire bien connu. Les patients sont informés et de la technique et du questionnaire auquel ils auront à répondre; cette information permet en effet une plus grande sensibilité de la mesure. ${ }^{7} \mathrm{Le}$ questionnaire relatif à ces stimuli auditifs est proposé au sujet le lendemain (Tableau I).

Chez les sujets intubés, deux observateurs indépendants ont estimé selon une cote l'intensité des fasciculations observées: une cote un, pour des fasciculations discrètes, une cote deux, pour des fasciculations grossières, et une cote trois, pour des fasciculations tellement importantes qu'elles provoquent la mobilisation d'une articulation.

Le temps d'éveil anesthésique était mesurẻ depuis la fin de l'anesthésie jusqu'au retour à un état de conscience déterminé par la réponse à des questions simples et par l'identification d'objets familiers.

Dans les suites post-anesthésiques, on mesurait de plus les données suivantes. D'abord de façon non spécifique à l'aide d'une question vague sur l'état général post-anesthésique, la présence de "courbatures" rapportée spontanément. Par la suite, de façon spécifique à l'aide de questions directes, la présence de nausées, de vomissements ou de mal de gorge, sans égard à l'intensité, la quantité ou la fréquence. De plus, les douleurs musculaires de type "postfasciculation" étaient quantifiées par un système de cotes, selon le nombre de régions corporelles impliquées: la cote un, pour une douleur de la nuque (ou les muscles de la face); la cote deux, pour les douleurs impliquant deux régions (tête, membres supérieurs, tronc, membres inférieurs); la cote trois, pour les douleurs impliquant trois régions.

Dans le but d'établir la présence ou l'absence de corrélation significative avec les variables à l'étude, plusieurs autres variables connexes ont été colligées. Ainsi, l'âge, le poids, la taille, la scolarité, la durée du jeûne pré-anesthésique (évaluées depuis 0:0 heure), la durée de l'anesthésie, les doses de médicaments utilisés tant en prémédication qu'à l'induction.

\section{Schéma expérimental}

Les trois facteurs contrôlés de cette ex périence sont les suivants: l'agent d'induction, la prémédication et la curarisation avec intubation. Chacun de ces facteurs est considéré à deux niveaux: deux médicaments pour la prémédication (diazépam et mépéridine), deux médicaments pour l'induction (diazépam et thiopental) et absence ou présence de curarisation. Les huit combinaisons possibles $(2 \times 2 \times 2)$ de ces conditions expérimentales définissent huit sous-groupes de cinq sujets chacun (Tableau II). De cette façon, les niveaux des facteurs sont équilibrés les uns par rapport aux autres.

\section{Mesures de contrôle}

Les sujets ont été attribués au hasard aux huit sous-groupes du plan. Ils ont tous été soumis aux mêmes procédures de base et les observateurs ont toujours été les mêmes. L'utilisation de la canule à trois entrées pour l'administration de l'agent d'induction permet d'éviter la dilution de 
TABLEAU II

Retension MNemoniQue Cotes Moyennes

\begin{tabular}{lllll}
\hline \hline & & \multicolumn{2}{c}{ Induction } \\
\cline { 4 - 5 } & Premedication & Intubation & Diazepam & Thiopental \\
\hline \multirow{2}{*}{ Dotaux } & présence & $5.40(5)^{*}$ & $6.40(5)$ \\
& Meperidine & absence & $5.40(5)$ & $5.60(5)$ \\
& Premedication & absence & $6.20(5)$ & $7.80(5)$ \\
& Diazepam: $5.70(20)^{*}$ & Présence: $6.45(20)$ & $6.90(5)$ & $7.60(5)$ \\
& Meperidine: $7.10(20)$ & Absence: $6.35(20)$ & Diazepam: $5.95(20)$ \\
& & Thiopental: 6.85(20) & Thduction \\
\hline
\end{tabular}

*Les chiffres indiquent la moyenne et les chiffres entre parenthèses le nombre de sujets du groupe ou sous-groupe.

la substance et le délai du maximum d'action, comme le suggèrent Egbert et Mitchell. ${ }^{8}$ Le double-insu fut respecté pour la prémédication et l'induction mis non pour l'intubation. Toutes les observations sont colligées par une mème infirmière de recherche qui ne connaît pas la médication d'induction et de prémédication. De plus, l'anesthésiste responsable du cas participe comme deuxième observateur à la mesure des fasciculations; il opère aussi à double-insu ne connaissant ni le médicament d'induction ni l'agent de prémédication (un deuxième anesthésiste a donné à sa place l'agent inducteur). Les données de mesures, tant subjectives qu'objectives, sont quantifiées et soumises à l'analyse statistique correspondant au schéma expérimental.

\section{Analyse statistique}

Les résultats furent traités par analyse de variance tenant compte des trois facteurs et de leurs interactions. ${ }^{9}$ De plus, les données qualitatives de type binaire (absence - présence) furent soumises à l'analyse logistique, ${ }^{10-11}$ analyse spécifique pour ce type de données qui tient également compte du schéma.

Les facteurs non contrôlés (données connexes) ont d'abord été analysés de la même façon, afin de vérifier l'homogénéité des groupes à leur égard. Ensuite on a recherché la corrélation entre ces variables et les variables expérimentales.

\section{RÉSUltats}

\section{Amnésie}

Le diazépam donné en prémédication a un effet amnésique significativement plus grand que celui de la mépéridine $(p<0.05)$. Les cotes de réten- tion mnémonique ont été respectivement de 5.7 et de 7.1 (Figure 1 et Tableau II).

Quant à l'induction, la cote du diazépam est inférieure (5.95) à celle du thiopental $(6.85)$ et l'épreuve de signification n'indique qu'une "tendance" à distinguer les effets des deux médicaments $(p<0.14)$. L'effet lié à l'intubation n'est pas significatif de même que celui lié aux diverses interactions entre les facteurs étudiés.

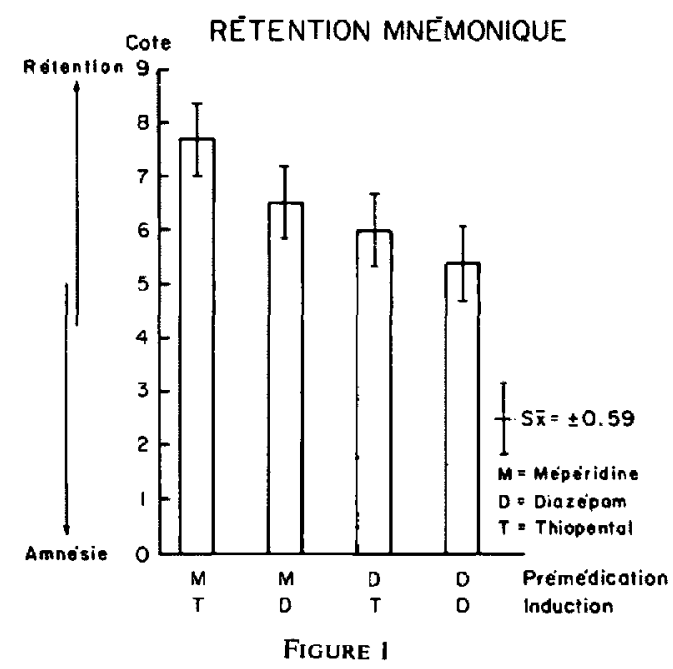

Mal de gorge

Comme on pouvait s'y attendre, plus de patients intubés ont eu mal à la gorge que de patients non intubés ( $p<0.0003$, Tableaux III et IV). Par ailleurs, le diazépam donné en induction diminue significativement l'incidence de mal de gorge ( 3 sur 20), par rapport au thiopental ( 8 sur 20$)-p<$ 0.05 . Les agents utilisés en prémédication ne se distinguent pas de façon significative. 
TABLEAU III

INCIDENCE DE MAL DE GORGE

\begin{tabular}{|c|c|c|c|c|}
\hline & \multirow[t]{2}{*}{ Premedication } & \multirow[t]{2}{*}{ Intubation } & \multicolumn{2}{|c|}{ Induction } \\
\hline & & & Diazepam & Thiopental \\
\hline & Diazepam & $\begin{array}{l}\text { Présence } \\
\text { Absence }\end{array}$ & $\begin{array}{l}1 / 5 \\
0 / 5\end{array}$ & $\begin{array}{l}3 / 5 \\
0 / 5\end{array}$ \\
\hline & Meperidine & $\begin{array}{l}\text { Présence } \\
\text { Absence }\end{array}$ & $\begin{array}{l}2 / 5 \\
0 / 5\end{array}$ & $\begin{array}{l}4 / 5 \\
1 / 5\end{array}$ \\
\hline \multirow[t]{2}{*}{ Totaux } & Premedication & Intubation & \multicolumn{2}{|c|}{ Induction } \\
\hline & $\begin{array}{l}\text { Diazepam: } 4 / 20 \\
\text { Meperidine: } 7 / 20\end{array}$ & $\begin{array}{l}\text { Présence: } 10 / 20 \\
\text { Absence: } 1 / 20\end{array}$ & \multicolumn{2}{|c|}{$\begin{array}{l}\text { Diazepam: } 3 / 20 \\
\text { Thiopental: } 8 / 20\end{array}$} \\
\hline
\end{tabular}

TABLEAU IV

ANAlyse Logistique - Mal de Gorge

\begin{tabular}{lcccc}
\hline \hline & ${ }^{*} \mathrm{~L} 1$ & $\mathrm{dl}$ & $\chi^{2}$ & \multicolumn{1}{c}{$\mathrm{P}$} \\
\hline Prémédication & 0.93 & 1 & 1.85 & 0.170 \\
Induction & 2.35 & 1 & 4.70 & 0.028 \\
Intubation & 6.58 & 1 & 13.18 & 0.0004 \\
Intéraction & 0.48 & 4 & 0.96 & n.s. \\
\hline
\end{tabular}

${ }^{*} \mathrm{LI}=$ logarithme de la vraisemblance maximale.

TABLEAU $V$

\begin{tabular}{lccc}
\hline & \multicolumn{2}{c}{ Induction } & \\
\cline { 2 - 3 } Autres effets secondaires & Diazepam & Thiopental & Difference \\
\hline Nausees & $9 / 20$ & $2 / 20$ & $\mathbf{p}<0.01$ \\
Vomissements & $4 / 20$ & $0 / 20$ & $\mathrm{p}<0.02$ \\
Courbatures & $11 / 20$ & $10 / 20$ & n.s. \\
Douleurs post-fasciculation & $7 / 20$ & $9 / 20$ & n.s. \\
\hline
\end{tabular}

Autres effets secondaires

Utilisé comrıe agent d'induction, le diazépam provoque plus de nausées et de vomissements que le thiopental (Tableau V). Il n'y a pas de différence significative entre ces deux médicaments pour la prévention des courbatures et des douleurs post-fasciculation (Tableau V). L'analyse des divers symptômes du Tableau $V$ ne révèle pas de différence significative entre les agents de prémédication; il n'y a pas non plus de différence entre les sujets intubés et les autres.

\section{Variables connexes}

Le diazépam, donné à l'induction, prolonge de façon significative le temps d'éveil (19 minutes par rapport à 11 minutes avec le thiopental - p < 0.01 ). La durée de prémédication (le temps entre la prémédication et le début de l'anesthésie) varie de façon hautement significative selon l'agent d'induction; elle est de 65.5 minutes pour le diazépam et de 40.9 minutes pour le thiopental $(p<0.01)$.

La dose de diazépam en induction est significativement plus élevée chez les sujets recevant le diazépam en prémédication que chez ceux recevant la mépéridine $(41.5 \mathrm{mg}$ versus $31.0 ; \mathrm{p}<$ 0.02). La durée moyenne de l'anesthésie a été de 35.7 minutes chez les patients intubés et de $\mathbf{4 0 . 6}$ minutes chez les non-intubés; cette différence significative du point de vue statistique n'a pas d'intérêt clinique. Il en va de même de la durée du jeûne: 11.8 heures chez ceux ayant reçu le diazépam en prémédicament et 10.5 heures avec la mépéridine.

L'analyse de variance ne démontre aucune différence significative entre les sous-groupes pour les facteur suivants: âge, poids, taille. scolarité, période de temps entre la fin de 
l'anesthésie et la réponse au questionnaire, doses de diazépam (en prémédication), de mépéridine, de thiopental, de succinylcholine.

\section{Discussion}

Le double emploi du diazépam, en prémédication et à l'induction, produit un effet amnésique des plus désirable. L'amnésie semble augmenter avec l'utilisation du diazépam comme agent inducteur, mais elle se manifeste nettement en prémédication (Figure 1). La cote moyenne de rétention mnémonique diminue de 7.7 à 6.5 en substituant le diazépam au thiopental (mépéridine en prémédication). L'effet du diazépam est plus important encore en prémédication: la cote moyenne est de 6.0 (thiopental à l'induction). Finalement, la cote est à son plus bas, à 5.4 , avec l'utilisation double du diazépam.

Il est intéressant de noter que, chez une population semblable de sujets informés, la cote moyenne obtenue dans des circonstances semblables avec la mépéridine et le thiopental était identique dans une expérience effectuée deux ans auparavant.' A cette cote de 7.7, le score maximal étant de neuf, les sujets se rappellent à peu près tout des stimuli auditifs présentés: à 5.4 (diazépam, diazépam), étant donné la difficulté progressive du questionnaire, les sujets se souviennent de la présence et de la nature des stimuli, mais ils errent dans leur remémoration de l'ordre de présentation ou de l'identification exacte.

Malgré l'attribution au hasard des différents médicaments, nous avons obtenu une variation importante de la durée de prémédication selon l'agent d'induction. L'influence de cette variation est difficile à estimer. L'induction s'est effectuée près du maximum d'action de la prémédication dans les deux cas. S`il y a influence, elle défavorise probablement l'addition d'effet diazépam-diazépam.

L'augmentation de la dose de diazépam pour l'induction chez les sujets prémédiqués au diazépam n'explique pas la différence entre la mépéridine et le diazépam en prémédication, puisque cette différence existe aussi chez les sujets induits au thiopental. Il est cependant possible que cette augmentation ait contribué à Jaddition d'effet diazépam-diazépam. Peu importe cette contribution théorique puisque l'on n'utilise pas une dose fixe de diazépam à l'induction, et que seul compte le résultat obtenu sur l'amnésie pour un même niveau d'induction anesthésique.

\section{Conclusions}

Dans nos conditions expérimentales, la prémédication au diazépam par voie intramusculaire produit une plus grande amnésie antérograde que la mépéridine. L'amnésie antérograde due au diazépam est bien connue $e^{12-13}$ mais uniquement lors d'une administration intraveineuse et non dans les circonstances habituelles de la prémédication en clinique. Selon les critères utilisés dans cette étude, le diazépam semble supérieur à la mépéridine en prémédication parce qu'il provoque une plus grande amnésie sans produire d'effet indésirable.

L'amnésie rêtrograde n'a jamais été démontrée comme un effet de quelque médicament que ce soit employé aux doses cliniques. ${ }^{13}$ Pourtant nos résultats semblent indiquer que l'amnésie du diazépam puisse s'étendre aux quelques minutes qui précèdent l'induction anesthésique. Même si la probabilité d'erreur (14 pour cent) nous empêche de conclure avec certitude, cette observation suggère qu'une plus grande sensibilité de la mesure pourrait mettre en évidence une amnésie rétrograde médicamenteuse.

L'emploi du diazépam à la fois en prémédication et en induction favorise l'amnésie. Le diazépam comme agent d'induction diminue la fréquence du mal de gorge dû à l'intubation (ce qui pourrait être expliqué par un effet prolongé de type myo-résolutif). Par contre, en comparaison au thiopental, le diazépam allonge le temps d'éveil et augmente la fréquence des nausées et des vomissements.

\section{RÉSUMÉ}

Dans cette étude à double-insu, l'effet du diazépam $(0.15 \mathrm{mg} / \mathrm{kg}$ I.M. en prémédication et $0.3 \mathrm{mg} / \mathrm{sec} \mathrm{I}$.V. en induction) sur la mémoire est comparé aux effets d'agents de prémédication et d'induction standards, la mépéridine ( $1 \mathrm{mg} / \mathrm{kg})$ et le thiopental $(2.5 \mathrm{mg} / \mathrm{sec})$. Quarante patients (32 femmes et 8 hommes), présentant un risque anesthésique de grade l et soumis à une chirurgie mineure élective, reçoivent un stimulus auditif immédiatement avant l'induction anesthésique. La rétention mnémonique et les effets désirables ou indésirables subjectifs sont évalués le lendemain. Les volontaires sont répartis au hazard en deux groupes recevant l'une ou l'autre prémédication. Chacun de ces groupes est de plus subdivisé en deux sous-groupes correspondant aux deux agents d'induction. L'absence ou la pré- 
sence d'intubation détermine un niveau ultérieur de subdivision aléatoire.

En prémédication, le diazépam produit plus d'amnésie antérograde que la mépéridine sans induire d'effets indésirables. Nos résultats laissent entrevoir la possibilité d'une amnésie rétrograde produite par le diazépam à l'induction. Les effets sur la mémoire sont plus grands lorsque le diazépam est utilisé à la fois en prémédication et à l'induction. Les maux de gorge de l'intubation sont moins fréquents avec le diazépam qu'avec le thiopental, mais le diazépam produit plus de nausées et de vomissements.

\section{SUMMARY}

In this double-blind study, the effects of diazepam $(0.15 \mathrm{mg} / \mathrm{kg}$ intramuscularly for premedication and $0.3 \mathrm{mg} / \mathrm{sec}$ intravenously for induction) on memory were compared to those of standard premedication and induction agents, meperidine (1 $\mathrm{mg} / \mathrm{kg}$ ) and thiopentone (2.5 $\mathrm{mg} / \mathrm{sec}$ ). Forty patients ( 32 females and 8 males), in physical status I, undergoing minor elective operations, received an auditory stimulus immediately before induction of anaesthesia; stimulus recall as well as subjective side-effects were assessed the following day. The volunteers were randomly assigned to either of two groups, each receiving one of the premedicaments. The two groups were further subdivided into sub-groups corresponding to the induction agents and intubation.

As a premedicant, diazepam produced more anterograde amnesia than meperidine without inducing any undesirable effect. Our results suggest that diazepam as an induction agent could possibly produce retrograde amnesia. The amnesic effects were more pronounced when diazepam was used successively for premedication and induction. Diazepam prevented the sore throat of intubation better than thiopentone, though it induced more nausea and vomiting.

\section{REMERCIEMENTS}

Ce travail a pu être réalisé en partie grâce à une subvention de la compagnie Hoffmann-La Roche du Canada.

\section{Bibliographie}

1. Dalen, J.E., Evans, G.L., Banas, J.S., Brooks, H.L., Paraskos, J.A., \& Dexter, L. The hemodynamic and respiratory effects of diazepam $\left(\right.$ Valium $\left.^{\mathrm{R}}\right)$. Anesthesiology 30: 259 (1969).

2. MCCLISH, A. Diazepam as an intravenous induction agent for general anaesthesia. Can. Anaes. Soc. J. 13:561 (1966).

3. Steen, S.N., Weitzner, S.W., Amaha, K., \& MARTINeZ, L.R. The effect of diazepam on the respiratory response to carbon dioxide. Can. Anaes. Soc. J. 13: 374 (1966).

4. MASPOLI, M. Le Valium ${ }^{R}$, son action sur la respiration. Schweiz. med. Wschr. 97: 320 (1967).

5. NorRIS, W. \& TELFER, A.B.M. Diazepam in cardioversion. N. Engl. J. Med. 273: 650 (1965).

6. BarRon, D.W. \& DundeE, J.W. Clinical studies of induction agents XVIl: Relationship between dosage and side effects of intravenous barbiturates. Brit. J. Anaesth. 39: 24 (1967).

7. Authier, L., Rousseau, P., Paquin, P., OuelLeTte, M., TAILlefer, J. JobiN, D., \& BarRY, P.P. Amnésie de mémoration post-anesthésique. I: Effet de l'information. Canad. Anaesth. Soc. J, $2 l$ : 46(1974)

8. Egbert, Lt. COMdR. L.D., \& Mitchell, Lt. ComDr. G.D. Technical problems of studying intravenous anesthetics in man. Anesthesiology 22: $186(1961)$.

9. WINER, B.J. Statistical principles in experimental design, 2nd ed. Montréal, McGraw-Hill (1971).

10. Cox. D.R. The analysis of binary data. London, Methuen (1970).

11. Gagnon, M.-A. Subjective phenomena in drug trials. International Journal of Clinical Pharmacology and Biopharmacy 15(4): 155 (1977).

12. Pandit, S.K., DUNdeE, J.W., \& KeILTY, S.R. Amnesia studies with intravenous premedication. Anaesthesia 26: 421 (1971).

13. Clarke, P.R.F., Eccersley, P.S., Frisay, J.P., \& THORNTON, J.A. The amnesic effect of diazepam (Valjum). Brit. J. Anaesth. 42: 690 (1970). 